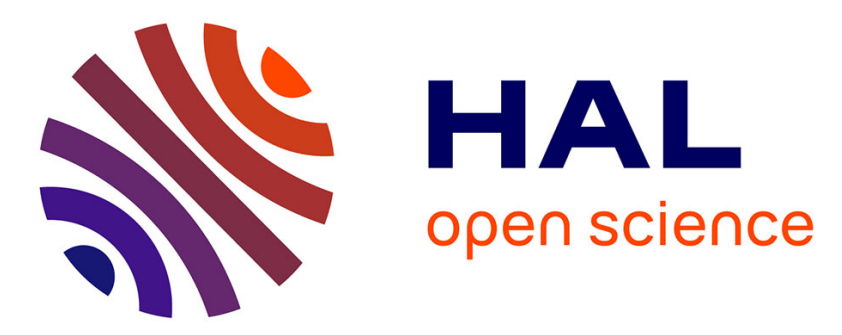

\title{
Radiative Electron Attachment and Photodetachment Rate Constants for Linear Carbon Chains
}

\author{
Miguel Lara-Moreno, Thierry Stoecklin, Philippe Halvick
}

\section{To cite this version:}

Miguel Lara-Moreno, Thierry Stoecklin, Philippe Halvick. Radiative Electron Attachment and Photodetachment Rate Constants for Linear Carbon Chains. ACS Earth and Space Chemistry, 2019, 3, pp.1556 - 1563. 10.1021/acsearthspacechem.9b00098 . hal-03044624

\section{HAL Id: hal-03044624 \\ https://cnrs.hal.science/hal-03044624}

Submitted on 14 Dec 2020

HAL is a multi-disciplinary open access archive for the deposit and dissemination of scientific research documents, whether they are published or not. The documents may come from teaching and research institutions in France or abroad, or from public or private research centers.
L'archive ouverte pluridisciplinaire HAL, est destinée au dépôt et à la diffusion de documents scientifiques de niveau recherche, publiés ou non, émanant des établissements d'enseignement et de recherche français ou étrangers, des laboratoires publics ou privés. 


\title{
Radiative electron attachment and
}

\section{photodetachment rate constants for linear carbon chains}

\author{
Miguel Lara-Moreno, Thierry Stoecklin, ${ }^{*}$ and Philippe Halvick \\ Université de Bordeaux, ISM, UMR 5255, 33405, Talence, France \\ E-mail: thierry.stoecklin@u-bordeaux.fr
}

\begin{abstract}
Since their recent detection in interstellar clouds and circumstellar envelopes, polyyne and cyanopolyyne anions have raised the question of their possible mechanisms of formation, destruction and excitation. These anions are observed in the same regions than the corresponding neutral species, with anion-to-neutral abundance ratios of a few percent. It is believed that the abundance ratios are controlled mainly by the radiative attachment processes. We present a quantum study of the radiative electron attachment and photodetachment rate constants for selected linear carbon-chain anions, namely the already detected interstellar anions as well as other potential candidates. The rate constants are calculated within the rigid molecule approximation, with the attached or ejected electron described by a plane wave. A qualitative agreement is obtained with the previous accurate quantum results. For the radiative electron attachment process, the discrepancies between the quantum rate constants calculated here and the statistical rate constants currently used in astronomical models are relatively small for the shortest carbon chains, but increase strongly with the number of atoms in the chain.
\end{abstract}




\section{Keywords}

Interstellar anions, radiative electron attachment, photodetachment, polyynes, cyanopolyynes

\section{Introduction}

The idea of interstellar anions was born in the late twentieth century when several authors ${ }^{1-3}$ discussed their possible detection as well as their role in the formation of other interstellar molecules. However the discovery of the first interstellar anion came almost 30 years later when $\mathrm{C}_{6} \mathrm{H}^{-}$was detected. ${ }^{4}$ This opened the way to the subsequent detection of five further anions, namely $\mathrm{C}_{4} \mathrm{H}^{-5}, \mathrm{C}_{8} \mathrm{H}^{-6}, \mathrm{C}_{3} \mathrm{~N}^{-7}, \mathrm{C}_{5} \mathrm{~N}^{-8}$ and $\mathrm{CN}^{-9}$. These findings have led to a growing interest in the chemistry of the interstellar anions with a special emphasis on their mechanisms of formation and destruction.

So far, interstellar anions have been observed in the same regions of the circumstellar envelope of IRC +10216 and the dark molecular cloud TMC-1 than their neutral precursors and are then expected to be formed through radiative electron attachment (REA):

$$
A+e^{-} \rightarrow A^{-}+h \nu
$$

From the observed value of the anion-to-neutral abundance ratios and under the assumption that anions are mostly produced by REA, the REA rate constants at $300 \mathrm{~K}$ are assumed to be $9 \times 10^{-11}, 1.4 \times 10^{-8}, 2.5 \times 10^{-8}, 1.9 \times 10^{-9} \mathrm{~cm}^{3}$ molecule ${ }^{-1} \mathrm{~s}^{-1}$ for $\mathrm{C}_{4} \mathrm{H}^{-}, \mathrm{C}_{6} \mathrm{H}^{-}, \mathrm{C}_{8} \mathrm{H}^{-}$, and $\mathrm{C}_{3} \mathrm{~N}^{-}$, respectively. ${ }^{7}$ Dissociative attachment in which molecular fragmentation follows the primary electron attachment might in principle be also considered as a possible formation mechanism of the anions. However, this process can happen only if the sum of the collision energy and the electron affinity (EA) of the neutral precursor is larger than the dissociation energy of the anion. At low temperature, only few anions satisfy such requirement..

Experimental measurements of REA are very difficult mainly because an excellent vac- 
uum is required to prevent the stabilization of the anions through collisions with the background gas particles. For this reason most experimental measurement on electron attachment ${ }^{10-12}$ correspond to collisional stabilization rates. While there is no direct measurement of REA cross section or rate constant, there are nevertheless experimental data which suggest that REA rate constants can be large, up to $10^{-7} \mathrm{~cm}^{3}$ molecule ${ }^{-1} \mathrm{~s}^{-1}$. Indeed, experimental values of the lifetimes of metastable anions formed by electron attachment at very low pressure have been found to be large enough to allow radiative stabilization. ${ }^{13-16}$ Moreover in a recent work, the REA rate constant of $\mathrm{C}_{6}^{-}$has been extracted from a photodetachment experiment by applying the principle of detailed balance. ${ }^{17}$

On the theoretical side, Herbst ${ }^{3}$ suggested that for species with large EA, the REA coefficient could be close to $10^{-7} \mathrm{~cm}^{3}$ molecule ${ }^{-1} \mathrm{~s}^{-1}$ in the temperature range of molecular clouds, $10-50$ K. Later, Petrie ${ }^{18}$ and Petrie and Herbst ${ }^{19}$ derived an expression for the REA rate coefficients based on the Phase Space Theory (PST) in which it is assumed that the electron is attached through the formation of a temporary negative ion which then can be followed by autodetachment or stabilization by radiative emission. This approach was used to obtain REA rate coefficients for $\mathrm{CN},{ }^{18} \mathrm{C}_{3} \mathrm{~N},{ }^{19} \mathrm{C}_{n}{ }^{20}(n=4-9)$ and $\mathrm{C}_{n} \mathrm{H}^{21}(n=2-$ 8). It may be noted that the PST REA rate coefficient increases rapidly with the size of the molecule since the statistical autodetachment rate of the temporary anion is inversely proportional to the density of vibrational levels.

Recently, Douguet et al. ${ }^{22},{ }^{23}$, Khamesian et al. ${ }^{24}$ proposed a new theory of REA from a quantum mechanical point of view and suggested a new mechanism in which the electron is attached without the formation of any intermediate state. In order to distinguish between the two mechanisms, they called direct REA (DREA) the one-step process and indirect REA (IREA) the two-step process proposed by Herbst ${ }^{3}$. Their calculated DREA rates for some of the detected anions are several orders of magnitude smaller than the IREA rates calculated by Herbst and Osamura ${ }^{21}$. Furthermore, considering again a quantum-mechanical approach based on first principles for both steps of the indirect mechanism, Douguet et al. ${ }^{23}$ concluded 
that the contribution of IREA process to the formation of $\mathrm{CN}^{-}$could be negligible compared to that of the DREA process. They suggested it could be the case for any molecules if no unusual threshold effects, virtual states or vibrational Feshbach dipole resonances are present.

Photodetachment (PD) is the reverse process of REA. The cross sections of both processes are related by the principle of detailed balance. PD is expected to be one of the most important mechanisms of destruction of interstellar anions.

$$
A^{-}+h \nu \rightarrow A+e^{-}
$$

Indeed, Kumar et al. ${ }^{25}$ found that ultraviolet PD is the main destruction mechanism for both $\mathrm{CN}^{-}$and $\mathrm{C}_{3} \mathrm{~N}^{-}$anions in IRC +10216 . Experimental measurements of $\mathrm{PD}$ are more feasible and experimental data are available for some of the detected anions. ${ }^{25-27}$ On the theoretical side, many approaches have been also applied to the study of PD. ${ }^{24,28-30}$ The main differences among these methods are the treatment of the bound and continuum wavefunction of the outgoing electron.

In a previous work, ${ }^{30}$ we introduced a method that satisfactorily applied to the study of both DREA and PD for diatomic molecules, giving a good agreement with PD experiments. The method uses the first-order Born approximation (FBA) for the electron continuum wavefunction and Dyson orbitals to describe the electron bound wavefunction. In the present work, we apply this method to linear carbon-chain molecules in the rigid molecule approximation. We compute the DREA and PD rates for the detected interstellar anions as well as for other potential candidates.

\section{Methods}

A PD process (eq. 2) with absolute cross section $\sigma_{\mathrm{PD}}$ proceeds at rate

$$
k_{\mathrm{PD}}=4 \pi \int_{E_{\mathrm{EA}}}^{E_{\mathrm{H}}} J_{\lambda}(E) \sigma_{\mathrm{PD}}(E) d E
$$


where $J_{\lambda}(E)$ is the average intensity of the interstellar radiation field (ISRF) in units of photons $\mathrm{cm}^{-2} \mathrm{~s}^{-1} \mathrm{sr}^{-1} \mathrm{eV}^{-1}$. The integral over photon energy $E$ runs from the EA threshold of the neutral molecule $E_{\mathrm{EA}}$ to the hydrogen ionization limit $\mathrm{E}_{\mathrm{H}}=13.6 \mathrm{eV}$. Here, we use the Draine 1978 ISRF with extension, defined byHeays et al. ${ }^{31}$, based on the ISRF of Draine ${ }^{32}$ between 91.2 and $200 \mathrm{~nm}$, and with the extension of van Dishoeck and Black ${ }^{33}$ at longer wavelengths.

On the other hand, the rate constant for a REA process (eq. 1) with cross section $\sigma_{\text {REA }}$ is

$$
k_{\mathrm{REA}}(T)=\sqrt{\frac{8}{m_{e} \pi}}\left(k_{\mathrm{B}} T\right)^{-3 / 2} \int_{0}^{\infty} \sigma_{\mathrm{REA}}(\epsilon) \mathrm{e}^{-\epsilon / k_{\mathrm{B}} T} \epsilon d \epsilon,
$$

where $k_{\mathrm{B}}$ is the Boltzmann's constant, $m_{e}$ and $\epsilon=E-E_{\mathrm{EA}}$ are the electron mass and collision energy respectively. Owing to the electron-molecule mass ratio, we can assume that the collision energy is the energy of the electron. Therefore, the temperature in eq. 4 is the electron temperature. The total REA cross section is the sum of the contributions from the DREA and IREA processes. Based on the result of Douguet et al. ${ }^{23}$, we will neglect the contribution from IREA and make the assumption that $\sigma_{\mathrm{REA}} \simeq \sigma_{\mathrm{DREA}}$.

The use of equations (3) and (4) requires the knowledge of PD and DREA cross sections respectively. Both cross sections are related by the principle of detailed balance, ${ }^{34}$ which can be expressed by a simple relation when the rovibrational motion is neglected:

$$
\frac{\sigma_{\mathrm{DREA}}}{\sigma_{\mathrm{PD}}}=\frac{g_{a}}{g_{n}} \frac{E^{2}}{2 m_{e} c^{2} \epsilon},
$$

where $c$ is the speed of light, $g_{a}$ and $g_{n}$ are the statistical weights of the anion and the neutral molecule, respectively. Equation (5), which is also known as the Milne relation, ${ }^{35}$ reduces the computational effort because knowing $\sigma_{\mathrm{PD}}$, we can obtain $\sigma_{\mathrm{DREA}}$ and vice versa. Therefore we will focus only on the calculation of $\sigma_{\mathrm{PD}}$.

For a randomly oriented ensemble of molecules, the cross section $\sigma_{\mathrm{PD}}$ can be written 
$\operatorname{as}^{36,37}$

$$
\sigma_{\mathrm{PD}}(E)=\frac{4 \pi^{2} e^{2} E}{9 \hbar c} \sum_{l m} \sum_{\kappa}\left\|d_{l m}^{\kappa}\right\|^{2},
$$

where the summation indexes run over the outgoing electron orbital angular momentum quantum number $l$, its projection onto the body-fixed $z$ axis $m$ and the component $\hat{\mu}_{\kappa}$ of the dipole operator . The matrix elements of the dipole operator are obtained as

$$
d_{l m}^{\kappa}=N_{0}^{\frac{1}{2}}\left\langle\Psi_{l m}\left|\hat{\mu}_{\kappa}\right| \Psi_{d}\right\rangle
$$

where $N_{0}$ is the product of the spin and electronic degeneracy factor, ${ }^{30} \Psi_{l m}(\mathbf{r})$ is the wavefunction of the outgoing electron and $\Psi_{d}(\mathbf{r})$ is the Dyson orbital. Let us recall that the Dyson orbital $^{38,39}$ is a molecular orbital which represents the change of the molecular electronic configuration induced by electron attachment or photodetachment. In this study we employ the FBA, thus representing $\Psi_{l m}$ by a plane wave.

An important ingredient of the calculation of the matrix elements of the dipole operator is the use of the orthogonality between the continuum wavefunction and the Dyson orbital. This relationship avoids a spurious dependence of equation (7) on the choice of the origin of the coordinate system as well as an improper behaviour of the cross section as a function of energy near threshold. ${ }^{37}$ The orthogonality between the continuum wavefunction and the Dyson orbital was then enforced by using Gram-Schmidt orthogonalization, as detailed in the Supporting Information.

The Dyson orbitals have been extracted from the ground state CASSCF wavefunctions of the anions and the corresponding neutral molecules. ${ }^{30}$ If $\psi^{N}\left(\vec{r}_{1}, \vec{r}_{2}, \ldots, \vec{r}_{N}\right)$ represents the electronic wavefunction of the $N$-electron molecular anion and $\psi^{N-1}\left(\vec{r}_{1}, \vec{r}_{2}, \ldots, \vec{r}_{N-1}\right)$ the one of the $(N-1)$-electron neutral molecule, then the Dyson orbital is obtained by computing the $(N-1)$-dimensional integral ${ }^{30}$

$$
\psi^{\text {Dyson }}(\vec{r})=\sqrt{N} \int \psi^{N-1}\left(\vec{r}_{1}, \ldots, \vec{r}_{N-1}\right) \psi^{N}\left(\vec{r}_{1}, \ldots, \vec{r}_{N}\right) d \vec{r}_{1} \ldots d \vec{r}_{N-1}
$$


The symmetry of the Dyson orbitals is of particular relevance for the calculation of the matrix elements of the dipole operator (equation 7). In section 3.2, we discuss the relation between the Dyson orbital symmetry and the REA rate constant at low temperature.

The electronic wavefunctions of both neutral and ionic species have been calculated with the single-state CASSCF method. We used the aug-cc-pVnZ basis sets restricted to the $d$ maximum angular momentum, with $n=5$ for the molecules having ten atoms or more, and $n=6$ for other molecules. This choice was guided by the need of using very diffuse orbitals for obtaining a reliable description of the anions while keeping the total number of atomic orbitals under a reasonable limit. DREA and PD cross sections are dependent on the size of the basis set. In a previous work ${ }^{30}$, we investigated the variations of the PD cross sections versus the size of the basis set for three diatomic anions. Relative differences of a few percent and up to $25 \%$, depending on the molecule and the collision energy, were observed.

For diatomic, triatomic, and $\mathrm{C}_{3} \mathrm{H}$ molecules, we used the valence active space. For larger molecules, the active space was taken smaller than the valence active space, with a maximum of 15 electrons in 14 molecular orbitals. The molecular geometries used in the CASSCF calculations for the Dyson orbitals were previously obtained at the same ab initio level from the geometry optimisation of the anions. For $\mathrm{CN}^{-}$, the experimental geometry was used. All electronic calculations were performed with the MOLPRO package. ${ }^{40}$ For selected neutral and ionic species, the electron affinities, dipole moments and equilibrium geometries calculated with the CASSCF method defined above are collected in five tables (see Supporting Information). These results are in qualitative agreement with available experimental data and prior theoretical results. 
Table 1: Calculated PD rate coefficients in $\mathrm{s}^{-1}$ for selected linear molecular anions. The format $a(b)$ correspond to $a \times 10^{b}$

\begin{tabular}{|c|c|c|c|c|}
\hline \multirow{2}{*}{$n$} & \multicolumn{2}{|r|}{$\mathrm{C}_{n}^{-}$} & \multicolumn{2}{|c|}{$\mathrm{C}_{n} \mathrm{H}^{-}$} \\
\hline & This work & Millar et al. ${ }^{41}$ & This work & Millar et al. ${ }^{41}$ \\
\hline 1 & & & $3.32(-8)$ & $1.41(-8)$ \\
\hline 2 & $4.02(-9)$ & $3.77(-9)$ & $4.00(-9)$ & $3.39(-9)$ \\
\hline 3 & $4.18(-9)$ & $6.60(-9)$ & $8.75(-9)$ & $7.50(-9)$ \\
\hline 4 & $5.84(-9)$ & $2.16(-9)$ & $4.67(-9)$ & $2.50(-9)$ \\
\hline 5 & $4.42(-9)$ & $3.41(-9)$ & $8.38(-9)$ & $4.35(-9)$ \\
\hline 6 & $7.83(-9)$ & $1.90(-9)$ & $5.41(-9)$ & $2.23(-9)$ \\
\hline 7 & $5.18(-9)$ & $2.75(-9)$ & $8.08(-9)$ & $3.39(-9)$ \\
\hline 8 & $1.05(-8)$ & $1.67(-9)$ & $5.96(-9)$ & $2.08(-9)$ \\
\hline 9 & $5.47(-9)$ & $2.36(-9)$ & $7.48(-9)$ & $2.87(-9)$ \\
\hline 10 & $1.39(-8)$ & $1.67(-9)$ & $7.12(-9)$ & $2.13(-9)$ \\
\hline \multirow{2}{*}{$n$} & \multicolumn{2}{|c|}{$\mathrm{C}_{n} \mathrm{~N}^{-}$} & \multicolumn{2}{|c|}{$\mathrm{HC}_{n} \mathrm{O}^{-}$} \\
\hline & This work & Millar et al. ${ }^{41}$ & This work & Millar et al. ${ }^{41}$ \\
\hline 1 & $5.10(-9)$ & $2.17(-9)$ & $8.48(-8)$ & $1.38(-7)$ \\
\hline 2 & $4.92(-9)$ & $3.86(-9)$ & & 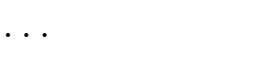 \\
\hline 3 & $3.90(-9)$ & $1.80(-9)$ & & $\cdots$ \\
\hline 4 & $5.14(-9)$ & $3.14(-9)$ & & \\
\hline 5 & $5.03(-9)$ & $1.70(-9)$ & & \\
\hline 6 & $6.44(-9)$ & $2.74(-9)$ & & . \\
\hline 7 & $4.90(-9)$ & $1.53(-9)$ & & . . \\
\hline
\end{tabular}

\section{Results}

\subsection{Photodetachment rates}

PD cross sections required for the calculation of the rate coefficients, equation (3), were calculated by neglecting the contributions from excited states of the neutral molecule. The inclusion of such contributions is expected to increase the PD rate coefficients, so our calculated rates should be considered as a lower limit. The calculated PD rates for selected linear anions are shown in Table 1. The essential result is that all the rates are found to be of the same order of magnitude $\left(10^{-9}-10^{-8}\right)$, regardless of the anion family and the carbon chain size.

Table 1 gives also the PD rate coefficients obtained by estimating the PD cross sections 

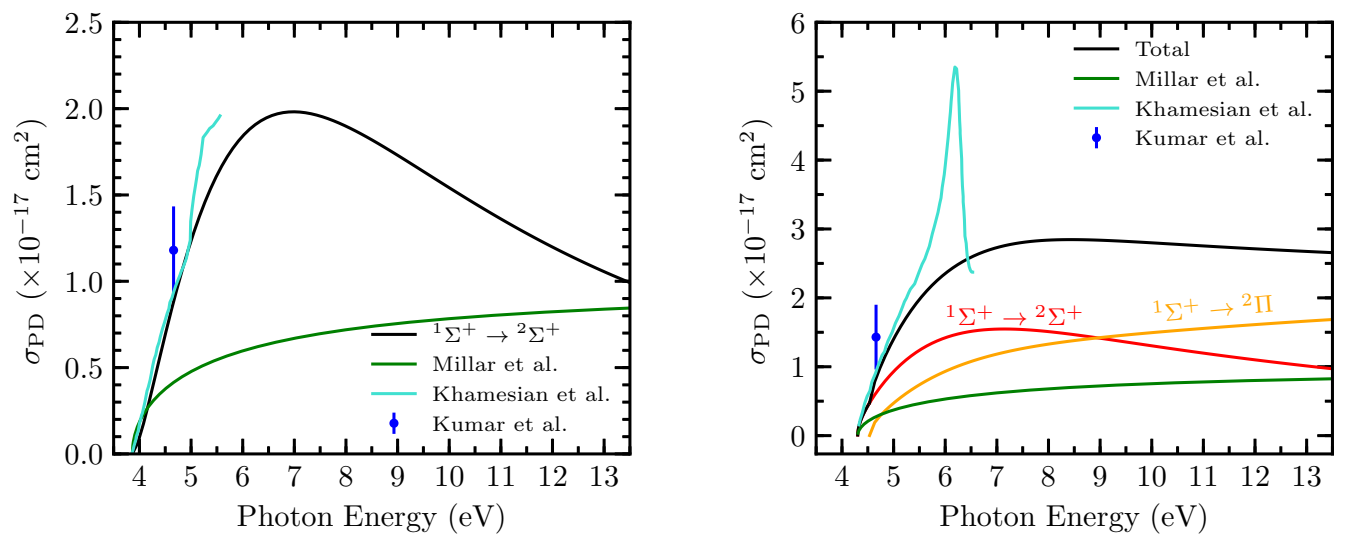

Figure 1: Photodetachment cross sections of $\mathrm{CN}^{-}$(left panel) and $\mathrm{C}_{3} \mathrm{~N}^{-}$(right panel).

with the simple empirical formula:

$$
\sigma_{\mathrm{PD}}=\sigma_{\infty}\left(1-\frac{E_{\mathrm{EA}}}{E}\right)^{1 / 2}
$$

where $\sigma_{\infty}$ is the PD cross section as $E \rightarrow \infty$, usually considered equal to $10^{-17} \mathrm{~cm}^{2}$. The formula (9), which was initially proposed by Millar et al. ${ }^{41}$, has been widely used to obtain the current $\mathrm{PD}$ rates included in many astrochemical databases (KIDA $\left.{ }^{1}, \mathrm{UMIST}^{2}\right)$. Comparing both results, we can conclude that the currently used PD rates are underestimated by approximately a factor of 3 . The main reason for such differences is due to the fact that equation (9) not only underestimates the PD cross sections but also does not reproduce the energy dependence especially at low photon energy, as shown by Figure 1.

The FBA photodetachment cross sections are also compared in Figure 1 to the accurate quantum results of Khamesian et al. ${ }^{24}$ for $\mathrm{CN}^{-}$and $\mathrm{C}_{3} \mathrm{~N}^{-}$. A good agreement is observed for collision energy smaller than $\sim 1 \mathrm{eV}$ above the threshold. For $\mathrm{CN}^{-}$, the discrepancy above $5 \mathrm{eV}$ comes from the opening of the $A^{2} \Pi$ excited state of $\mathrm{CN}$ which is not included in the FBA calculation. For $\mathrm{C}_{3} \mathrm{~N}^{-}$, a resonance is shown at $6 \mathrm{eV}$ in the accurate quantum PD cross section, but not in the FBA cross section since resonances can not be obtained in the FBA.

The replacement of the currently used PD rate coefficients by those calculated in the

\footnotetext{
${ }^{1}$ http: //kida.obs.u-bordeaux1.fr

${ }^{2}$ http: //www.udfa.ajmarkwick.net
} 
present work could lead to a decrease of the anion abundances predicted by the astrochemistry models. However, in the astrochemistry models, the photodetachment rates need to be multiplied by the attenuation factor which depends on the extinction. In the dark cloud TMC-1, extinction is assumed to be large. ${ }^{42}$ Then photodetachment is not a major destruction process of anions. Other destruction processes are considered, such as associative detachment and neutralization with cations. ${ }^{43}$ In contrast it has been shown ${ }^{25}$ that UV photodetachment is the most important destruction mechanism of anions in the circumstellar envelope of IRC +10216 where the extinction is low.

\subsection{Radiative electron attachment rates}

The calculated DREA rate coefficients obtained from the PD cross sections by the principle of detailed balance at selected temperatures are shown in Table 2. As the contributions from the excited states of the neutral molecule were neglected in the calculations of the PD cross sections, they are not taken into account in the DREA computations, either. This assumption is expected to have little influence on the DREA rates since at low temperatures only the electronic ground state of the neutral molecules should be populated.

The calculated DREA rate coefficients are seen to be small, ranging from $10^{-17}$ to $10^{-14}$ $\mathrm{cm}^{3}$ molecule $\mathrm{s}^{-1}$. A qualitative agreement is observed with the more accurate quantum calculations, based on the Kohn variational principle $(\mathrm{KVP})$, for $\mathrm{CN}, \mathrm{C}_{5} \mathrm{~N}, \mathrm{C}_{2} \mathrm{H}$ and $\mathrm{C}_{4} \mathrm{H}$.

For many molecules in Table 2, the rate coefficients are also found to not exhibit any temperature dependence. The very low temperature temperature regime is well understood on the basis of Wigner threshold laws. ${ }^{44}$ The DREA cross section at threshold can be derived from the detailed balance principle and the PD cross section at threshold ${ }^{37}$ obtained in the FBA which neglect the interaction between the neutral molecule and the incident electron:

$$
\sigma_{\mathrm{DREA}}(\epsilon \rightarrow 0)=\sum_{l} a_{l} \epsilon^{l-1 / 2}
$$


Table 2: Calculated DREA rate coefficients in $\mathrm{cm}^{3}$ molecule ${ }^{-1} \mathrm{~s}^{-1}$ for selected linear molecules for various temperatures. The symmetry type of the Dyson orbital is given in the column $\Psi_{d}$. The format $a(b)$ correspond to $a \times 10^{b}$

\begin{tabular}{|c|c|c|c|c|c|c|}
\hline \multirow{2}{*}{ species } & \multirow{2}{*}{$\Psi_{d}$} & \multicolumn{5}{|c|}{$k_{\mathrm{REA}}(T)$} \\
\hline & & $T=10 \mathrm{~K}$ & $T=30 \mathrm{~K}$ & $T=50 \mathrm{~K}$ & $T=100 \mathrm{~K}$ & $T=300 \mathrm{~K}$ \\
\hline $\mathrm{C}_{2}$ & $\sigma_{g}$ & $1.03(-17)$ & $3.11(-17)$ & $5.19(-17)$ & $1.04(-16)$ & $3.14(-16)$ \\
\hline $\mathrm{C}_{3}$ & $\pi_{g}$ & $5.23(-18)$ & $1.57(-17)$ & $2.63(-17)$ & $5.28(-17)$ & $1.61(-16)$ \\
\hline $\mathrm{C}_{4}$ & $\pi_{g}$ & $2.34(-17)$ & $7.01(-17)$ & $1.17(-16)$ & $2.33(-16)$ & $6.97(-16)$ \\
\hline $\mathrm{C}_{5}$ & $\pi_{u}$ & $6.34(-15)$ & $6.32(-15)$ & $6.31(-15)$ & $6.27(-15)$ & $6.14(-15)$ \\
\hline $\mathrm{C}_{6}$ & $\pi_{u}$ & $1.22(-14)$ & $1.22(-14)$ & $1.22(-14)$ & $1.21(-14)$ & $1.17(-14)$ \\
\hline $\mathrm{C}_{7}$ & $\pi_{g}$ & $4.04(-17)$ & $1.21(-16)$ & $2.01(-16)$ & $3.98(-16)$ & $1.15(-15)$ \\
\hline $\mathrm{C}_{8}$ & $\pi_{g}$ & $6.48(-17)$ & $1.93(-16)$ & $3.21(-16)$ & $6.34(-16)$ & $1.82(-15)$ \\
\hline $\mathrm{C}_{9}$ & $\pi_{u}$ & $6.92(-15)$ & $6.87(-15)$ & $6.82(-15)$ & $6.70(-15)$ & $6.32(-15)$ \\
\hline $\mathrm{C}_{10}$ & $\pi_{u}$ & $8.30(-15)$ & $8.22(-15)$ & $8.14(-15)$ & $7.95(-15)$ & $7.39(-15)$ \\
\hline $\mathrm{CH}$ & $\pi$ & $2.36(-15)$ & $2.36(-15)$ & $2.37(-15)$ & $2.38(-15)$ & $2.41(-15)$ \\
\hline $\mathrm{C}_{2} \mathrm{H}$ & $\sigma$ & $2.55(-16)$ & $\begin{array}{l}2.63(-16) \\
7.0(-17)^{a}\end{array}$ & $2.70(-16)$ & $2.89(-16)$ & $3.65(-16)$ \\
\hline $\mathrm{C}_{3} \mathrm{H}$ & $\pi$ & $1.58(-16)$ & $1.61(-16)$ & $1.64(-16)$ & $1.72(-16)$ & $2.02(-16)$ \\
\hline $\mathrm{C}_{4} \mathrm{H}$ & $\sigma$ & $2.77(-15)$ & $\begin{array}{l}2.78(-15) \\
2.0(-16)^{\mathrm{a}}\end{array}$ & $2.80(-15)$ & $2.83(-15)$ & $2.97(-15)$ \\
\hline $\mathrm{C}_{5} \mathrm{H}$ & $\pi$ & $1.87(-15)$ & $1.86(-15)$ & $1.86(-15)$ & $1.84(-15)$ & $1.80(-15)$ \\
\hline $\mathrm{C}_{6} \mathrm{H}$ & $\pi$ & $1.67(-15)$ & $1.67(-15)$ & $1.67(-15)$ & $1.66(-15)$ & $1.62(-15)$ \\
\hline $\mathrm{C}_{7} \mathrm{H}$ & $\pi$ & $3.87(-16)$ & $4.01(-16)$ & $4.15(-16)$ & $4.51(-16)$ & $5.85(-16)$ \\
\hline $\mathrm{C}_{8} \mathrm{H}$ & $\pi$ & $7.54(-16)$ & $7.63(-16)$ & $7.73(-16)$ & $7.95(-16)$ & $8.80(-16)$ \\
\hline $\mathrm{C}_{9} \mathrm{H}$ & $\pi$ & $1.38(-15)$ & $6.87(-15)$ & $1.36(-15)$ & $1.35(-15)$ & $1.30(-15)$ \\
\hline $\mathrm{C}_{10} \mathrm{H}$ & $\pi$ & $1.12(-15)$ & $1.11(-15)$ & $1.11(-15)$ & $1.11(-15)$ & $1.09(-15)$ \\
\hline $\mathrm{CN}$ & $\sigma$ & $1.30(-17)$ & $\begin{array}{l}3.51(-17) \\
7.3(-16)^{\mathrm{b}}\end{array}$ & $5.71(-17)$ & $1.12(-16)$ & $3.31(-16)$ \\
\hline $\mathrm{C}_{2} \mathrm{~N}$ & $\pi$ & $1.31(-15)$ & $1.31(-15)$ & $1.32(-15)$ & $1.33(-15)$ & $1.37(-15)$ \\
\hline $\mathrm{C}_{3} \mathrm{~N}$ & $\sigma$ & $4.68(-15)$ & $4.70(-15)$ & $4.71(-15)$ & $4.75(-15)$ & $4.89(-15)$ \\
\hline $\mathrm{C}_{4} \mathrm{~N}$ & $\pi$ & $1.41(-15)$ & $1.41(-15)$ & $1.41(-15)$ & $1.41(-15)$ & $1.39(-15)$ \\
\hline $\mathrm{C}_{5} \mathrm{~N}$ & $\sigma$ & $8.92(-15)$ & $8.93(-15)$ & $8.94(-15)$ & $8.96(-15)$ & $\begin{array}{l}9.07(-15) \\
7.5(-15)^{\mathrm{c}}\end{array}$ \\
\hline $\mathrm{C}_{6} \mathrm{~N}$ & $\pi$ & $8.22(-16)$ & $8.29(-16)$ & $8.37(-16)$ & $8.56(-16)$ & $9.27(-16)$ \\
\hline $\mathrm{C}_{7} \mathrm{~N}$ & $\pi$ & $2.34(-15)$ & $2.23(-14)$ & $2.35(-15)$ & $2.36(-15)$ & $2.40(-15)$ \\
\hline $\mathrm{HCO}$ & $\sigma$ & $1.64(-17)$ & $1.67(-17)$ & $1.71(-17)$ & $1.79(-17)$ & $2.17(-17)$ \\
\hline
\end{tabular}

where $l$ is the orbital angular momentum and $a_{l}$ is a proportionality constant. Insertion of equation (10) into equation (4) leads to the temperature dependence at threshold of the DREA rate coefficient:

$$
k_{\mathrm{DREA}}(T \rightarrow 0)=\sum_{l} b_{l} T^{l}
$$


which can be rewritten

$$
k_{\mathrm{DREA}}(T \rightarrow 0) \approx b_{\lambda} T^{\lambda}+O\left(T^{\lambda}\right)
$$

if we denote by $\lambda$ the lowest value of $l$ for which $b_{\lambda}$ is non zero. The value of $\lambda$ can be determined using the expansions of the Dyson orbital and of the continuum wavefunction (see equations S4 and S5 in Supporting Information) and the $\Delta l= \pm 1$ selection rule. In the cases of Dyson orbitals of symmetry $\sigma\left(l^{\prime}=0,1, \ldots\right), \pi\left(l^{\prime}=1,2, \ldots\right)$ or $\pi_{u}\left(l^{\prime}=1,3, \ldots\right)$, we then obtain $\lambda=0$. The threshold scattering is thus dominated by the electron $s$-wave. The threshold DREA rate is independent of temperature.

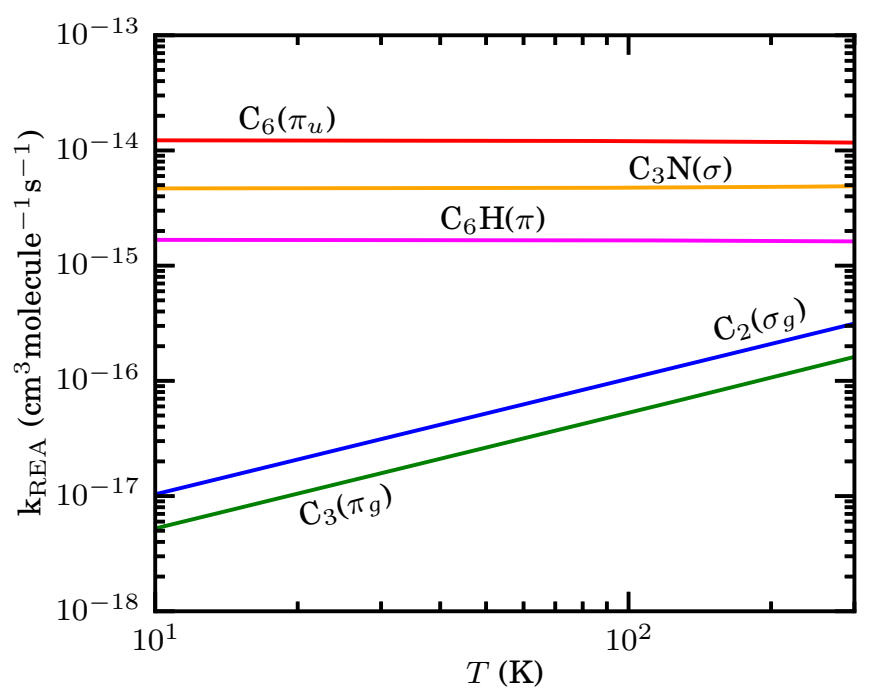

Figure 2: DREA rate coefficients for selected molecules. The symmetry of the Dyson orbital is indicated for each molecule.

On the other hand, in the cases of Dyson orbitals of symmetry $\sigma_{g}\left(l^{\prime}=0,2, \ldots\right)$ or $\pi_{g}$ $\left(l^{\prime}=2,4, \ldots\right)$, we obtain $\lambda=1$. The threshold scattering is dominated by the electron p-wave and the threshold DREA rate increases linearly with temperature.

Therefore, the DREA processes ruled by $p$-wave electrons do not occur when $T \rightarrow 0$ since $k_{\text {DREA }} \rightarrow 0$ while those ruled by $s$-wave electrons can occur since $k_{\text {DREA }} \rightarrow$ constant. Such behavior is explained by the presence or the absence of the centrifugal barrier $l(l+1) / r^{2}$. It is worth noting that tunneling through the centrifugal barrier is included in the FBA calculation since the solutions of the zero potential Hamiltonian are plane waves. 
The DREA rates calculated using equation 5 are represented for selected molecules in Figure 2 where the expected temperature dependence can be observed. By definition, the Wigner threshold law holds at threshold energy. For energies slightly above threshold, the cross section can be expanded in a power series in $k^{2}$ (the square of the scattering vector), the Wigner law being the first term of the series. As the energy increases, the Wigner law deviates progressively from reality. However, in the FBA, the interaction potential between the electron and the molecule is neglected. This makes the Wigner law valid for a large range of energies above threshold and then also for a large range of temperature as observed in Figure 2.

Below $50 \mathrm{~K}$, as shown by Figure 2, the rate coefficients of DREA processes driven by $p$-wave are lower by two order of magnitude then those driven by $s$-wave, even though the tunneling through the centrifugal barrier is taken into account for $p$-wave. DREA processes involving $p$-wave are then much more unlikely to occur than those involving $s$-wave at the low temperature range, 10-40 K, of the ISM regions where anions have been detected. Indeed, for the six anions already detected in the interstellar media, $\mathrm{CN}^{-}, \mathrm{C}_{3} \mathrm{~N}^{-}, \mathrm{C}_{5} \mathrm{~N}^{-}, \mathrm{C}_{4} \mathrm{H}^{-}$, $\mathrm{C}_{6} \mathrm{H}^{-}$, and $\mathrm{C}_{8} \mathrm{H}^{-}$, the DREA process is driven by s-wave scattering since the Dyson orbital is of $\sigma$ or $\pi$ symmetry as shown in Table 2 .

So far the REA rate coefficients used to model the formation of interstellar anion (KIDA, UMIST) have been calculated using the PST approach proposed by Petrie and Herbst ${ }^{19}$, see Table 3. This approach considers only the contribution from the IREA mechanism which is a combination of three elementary processes: first the formation of a temporary anion $\left(A^{-}\right)^{\dagger}$ in the continuum followed by autodetachment or stabilization by spontaneous emission:

$$
\mathrm{A}+e^{-} \underset{k_{\mathrm{d}}}{\stackrel{k_{\mathrm{c}}}{\rightleftarrows}}\left(\mathrm{A}^{-}\right)^{\dagger} \stackrel{k_{\mathrm{r}}}{\rightarrow} \mathrm{A}^{-}+h \nu
$$

In this approach, the autodetachment rate is controlled by the density of vibrational levels. A high density implies a low autodetachment rate thus allowing time for the radiative 
Table 3: REA rate constants at $T=300 \mathrm{~K}$, except for the KVP rate constants of $\mathrm{C}_{2} \mathrm{H}$ and $\mathrm{C}_{4} \mathrm{H}$ which are at $T=30 \mathrm{~K}$. In order to obtain the PST rate coefficients at different temperatures, the values must be multiplied by $(T / 300)^{1 / 2}$. The format $a(b)$ correspond to $a \times 10^{b}$.

\begin{tabular}{|c|c|c|c|}
\hline species & $\overline{\mathrm{PST}}$ & $\overline{\mathrm{FBA}}$ & KVP \\
\hline $\mathrm{C}_{4}$ & $1.4(-8)^{\mathrm{a}}$ & $6.97(-16)$ & \\
\hline $\mathrm{C}_{5}$ & $3.3(-8)^{\mathrm{a}}$ & $6.14(-15)$ & \\
\hline $\mathrm{C}_{6}$ & $1.7(-7)^{\mathrm{a}}$ & $1.17(-14)$ & \\
\hline $\mathrm{C}_{7}$ & $5.0(-7)^{\mathrm{a}}$ & $1.15(-15)$ & \\
\hline $\mathrm{C}_{8}$ & $1.7(-7)^{\mathrm{a}}$ & $1.82(-15)$ & \\
\hline $\mathrm{C}_{9}$ & $5.0(-7)^{\mathrm{a}}$ & $6.32(-15)$ & \\
\hline $\mathrm{C}_{2} \mathrm{H}$ & $2.0(-15) \mathrm{b}$ & $3.65(-16)$ & $7.0(-17)^{\mathrm{e}}$ \\
\hline $\mathrm{C}_{3} \mathrm{H}$ & $1.7(-14)^{b}$ & $2.02(-16)$ & \\
\hline $\mathrm{C}_{4} \mathrm{H}$ & $1.1(-8)^{b}$ & $2.97(-15)$ & $2.0(-16)^{\mathrm{e}}$ \\
\hline $\mathrm{C}_{5} \mathrm{H}$ & $4.1(-8) b$ & $1.80(-15)$ & \\
\hline $\mathrm{C}_{6} \mathrm{H}$ & $6.2(-8)^{b}$ & $1.62(-15)$ & \\
\hline $\mathrm{C}_{7} \mathrm{H}$ & $1.9(-7)^{b}$ & $5.85(-16)$ & \\
\hline $\mathrm{C}_{8} \mathrm{H}$ & $6.2(-8) \mathrm{b}$ & $8.80(-16)$ & \\
\hline $\mathrm{C}_{3} \mathrm{~N}$ & $2.6(-10)^{\mathrm{c}}$ & $4.89(-15)$ & \\
\hline $\mathrm{C}_{5} \mathrm{~N}$ & $1.3(-7)^{\mathrm{d}}$ & $9.07(-15)$ & $7.5(-15)^{\mathrm{f}}$ \\
\hline \multicolumn{4}{|c|}{ a Terzieva and Herbst ${ }^{20}$} \\
\hline \multicolumn{4}{|c|}{ b Herbst and Osamura ${ }^{21}$} \\
\hline \multicolumn{4}{|c|}{ c Petrie and Herbst ${ }^{19}$} \\
\hline \multicolumn{4}{|c|}{ d Cordiner and Millar ${ }^{45}$} \\
\hline \multicolumn{4}{|c|}{ e Douguet et al. ${ }^{23}$} \\
\hline \multicolumn{4}{|c|}{${ }^{f}$ Khamesian et al. ${ }^{24}$} \\
\hline
\end{tabular}

stabilization of the transient anion. In other words, as the number of atoms in the molecule increases, $k_{\mathrm{d}}$ decreases thus increasing $k_{\mathrm{REA}}$ up to to the limit $k_{\mathrm{REA}} \rightarrow k_{\mathrm{c}}$ for large molecules. The capture rate $k_{\mathrm{c}}$ can be approximated by

$$
k_{\mathrm{c}}(T)=\frac{\hbar^{2} g_{a}}{g_{n}} \sqrt{\frac{2 \pi}{m^{3} k T}}=\frac{g_{a}}{g_{n}}\left(\frac{T}{300}\right)^{-\frac{1}{2}} 4.982 \times 10^{-7} \mathrm{~cm}^{3} \text { molecule }{ }^{-1} \mathrm{~s}^{-1}
$$

This limit is almost reached by the largest molecules of Table 3 . If we compare the rate coefficients of Tables 2 and 3, we see that apart for the small molecules $\mathrm{C}_{2} \mathrm{H}$ and $\mathrm{C}_{3} \mathrm{H}$, they differ by several orders of magnitude. The main reason for these large discrepancies is the fact that the PST approach assumes that the electronic energy excess of the transient anion is 
efficiently transferred to the vibrational motions while our quantum approach is based on the rigid molecule approximation which means that no coupling is possible between the electronic and vibrational motions. As a matter of fact, there is no evidence yet that efficient transfer of energy between electronic and rovibrational motions can occur in electron-molecule collision.

The vibrational motion can be included in the quantum calculation of REA cross section with the help of the Franck-Condon approximation. Indeed, the electronic matrix elements of the transition dipole moment (TDM) defined in equation 7 weakly depend on the geometry of the molecule. ${ }^{23}$ Therefore the vibronic TDM can be obtained as a product of the electronic TDM for some fixed geometry by the overlap of the initial and final vibrational wavefunctions. Since the vibrational overlap is always smaller than 1 , the REA cross section then can not be enhanced by the vibrational motion.

On the other side, resonant processes due to rovibronic couplings are expected to occur in electron-molecules collision. Such resonant processes might generate the long lifetime transient anion which is the central assumption of the IREA model. A recent quantum treatment of the IREA process ${ }^{23}$, based on the approximate computation of the non-adiabatic vibronic coupling, has shown that IREA is negligible with respect to the DREA mechanism for the $\mathrm{CN} / \mathrm{CN}^{-}$system. The weakness of the IREA cross section was attributed by these authors to the small value of the overlap between the vibrational ground state of the neutral molecule and the highly excited vibrational state of the final anion as the EA is large ${ }^{24}$. Moreover, for a diatomic molecule such as CN, the density of vibrational levels is probably no large enough, as pointed out by Petrie ${ }^{18}$. For large molecules exhibiting low vibrational frequencies, the density of vibrational levels is expected to be very large, but again the EA is large and the excited vibrational levels of the anion at the EA energy should have a very small overlap with the vibrational ground state of the neutral molecule. There is nevertheless an interesting final case which is the one of the anions dipole bound states (DBS). Let us remind that DBS are weakly bound states where an electron is attached to a polar molecule by the charge-dipole long-range interaction. ${ }^{46}$ DBS are excited electronic states of anions. 
Since the energy and the geometry of the DBS state is very close to the energy and the geometry of the neutral precursor, then a good overlap of the vibrational functions can be expected. However, DBS concerns only the restricted class of molecules which exhibit a dipole moment larger than $\sim 2.5 \mathrm{D}$..

\section{Conclusion}

DREA and PD rate coefficients are calculated for the six detected anions and for other potential candidates belonging to the families $\mathrm{C}_{n}^{-}, \mathrm{C}_{n} \mathrm{H}^{-}, \mathrm{C}_{n} \mathrm{~N}^{-}$and also $\mathrm{HCO}^{-}$. Dyson orbitals and plane waves were respectively used to describe the bound wavefunction of the anions and the electron continuum wavefunction. A comparison between our PD rates and those which are currently in use on the astrochemical model shows that the current values always underestimate the PD rates. DREA rate coefficients are also obtained from the PD rates by using micro reversibility. For small molecules, the present DREA rates are found to be close to the statistical values, while for large molecules, they are smaller than the statistical REA rates by several order of magnitude. Therefore the present FBA approximate quantum approach of DREA rates cannot explain the formation of anion in ISM and this result is in agreement with other results obtained with the KVP more accurate quantum approach. Conversely, the statistical REA rates are in relatively good agreement with experiment and with the astronomical observations. But the validity of the statistical approach remain questionable and has not been checked against accurate calculations. On the other side, the experiments do not measure the REA rates, and the astronomical REA rates assume that REA is the single pathway to anion formation. Therefore the evidences for large REA rates remain weak.

For a few molecules, namely $\mathrm{CN}, \mathrm{C} 5 \mathrm{~N}, \mathrm{C} 2 \mathrm{H}$ and $\mathrm{C} 4 \mathrm{H}$, the DREA rate constants calculated with the KVP accurate quantum calculations are much smaller than the PST rates and than the rates deduced from astronomical observations. The FBA approximate quan- 
tum method has been applied here to a larger series of molecules. A qualitative agreement is obtained with the KVP DREA rate constants for the four molecules mentioned above, therefore demonstrating the reliability of the FBA calculations. In summary, one can state that the FBA calculations extend the KVP findings to a large series of molecules.

Besides DREA processes, the IREA processes should be also considered. Accurate quantum calculations of IREA are then needed while promising to be a tremendous task for large molecule as they will require using a realistic description of all the vibrational, rotational, continuum and bound electronic motions.

\section{Supporting information}

See supporting information for details of the calculation of the matrix elements of the dipole operator and for five tables comparing the electron affinities, dipole moments and equilibrium geometries calculated in this work with the available experimental data and prior theoretical results for selected neutral and anionic species.

\section{Acknowledgement}

This research have been supported by the Agence Nationale de la Recherche (Project ANRAnionCosChem). Computer time for this study was provided by the Mésocentre de Calcul Intensif Aquitain (MCIA), computing facilities of Université de Bordeaux and Université de Pau.

\section{References}

(1) Dalgarno, A.; McCray, R. A. The formation of interstellar molecules from negative ions. Astrophys. J. 1973, 181, 95-100. 
(2) Sarre, P. On the possible detection of negative ions in the interstellar medium. J. Chim. Phys. 1980, 77, 769-771.

(3) Herbst, E. Can negative molecular ions be detected in dense interstellar clouds? Nature 1981, 289, 656-657.

(4) McCarthy, M. C.; Gottlieb, C. A.; Gupta, H.; Thaddeus, P. Laboratory and astronomical identification of the negative molecular ion $\mathrm{C}_{6} \mathrm{H}^{-}$. Astrophys. J. 2006, 652, L141.

(5) Cernicharo, J.; Guélin, M.; Agúndez, M.; Kawaguchi, K.; McCarthy, M.; Thaddeus, P. Astronomical detection of $\mathrm{C}_{4} \mathrm{H}^{-}$, the second interstellar anion. Astron. Astrophys. 2007, 467, L37-L40.

(6) Brünken, S.; Gupta, H.; Gottlieb, C. A.; McCarthy, M. C.; Thaddeus, P. Detection of the carbon chain negative ion $\mathrm{C}_{8} \mathrm{H}^{-}$in TMC-1. Astrophys. J. 2007, 664, L43.

(7) Thaddeus, P.; Gottlieb, C. A.; Gupta, H.; Brünken, S.; McCarthy, M. C.; Agúndez, M.; Guélin, M.; Cernicharo, J. Laboratory and astronomical detection of the negative molecular ion $\mathrm{C}_{3} \mathrm{~N}^{-}$. Astrophys. J. 2008, 677, 1132.

(8) Cernicharo, J.; Guélin, M.; Agúndez, M.; McCarthy, M. C.; Thaddeus, P. Detection of $\mathrm{C}_{5} \mathrm{~N}^{-}$and vibrationally excited $\mathrm{C}_{6} \mathrm{H}$ in IRC +10216 . Astrophys. J. 2008, 688, L83.

(9) Agúndez, M.; Cernicharo, J.; Guélin, M.; Kahane, C.; Roueff, E.; Klos, J.; Aoiz, F. J.; Lique, F.; Marcelino, N.; Goicoechea, J. R.; González García, M.; Gottlieb, C. A.; McCarthy, M. C.; Thaddeus, P. Astronomical identification of $\mathrm{CN}^{-}$, the smallest observed molecular anion. Astron. Astrophys. 2010, 517, L2.

(10) Christophorou, L. G.; Olthoff, J. K. Electron attachment cross sections and negative ion states of $\mathrm{SF}_{6}$. Int. J. Mass Spec. 2001, 205, $27-41$. 
(11) Viggiano, A. A.; Miller, T. M.; Friedman, J. F.; Troe, J. Low-energy electron attachment to $\mathrm{SF}_{6}$. III. From thermal detachment to the electron affinity of $\mathrm{SF}_{6}$. J. Phys. Chem. 2007, 127, 244305.

(12) Knighton, W. B.; Miller, T. M.; Grimsrud, E. P.; Viggiano, A. A. Electron attachment to $\mathrm{PSCl}_{3}$. J. Chem. Phys. 2004, 120, 211-216.

(13) Foster, M. S.; Beauchamp, J. Electron attachment to sulphur hexafluoride: Formation of stable $\mathrm{SF}_{6}^{-}$at low pressure. Chem. Phys. Lett. 1975, 31, 482-486.

(14) Woodin, R. L.; Foster, M. S.; Beauchamp, J. L. Ion cyclotron resonance studies of radiative and dissociative electron attachment processes at low pressures. J. Chem. Phys. 1980, 72, 4223-4227.

(15) Graupner, K.; Field, T. A.; Saunders, G. C. Experimental Evidence for Radiative Attachment in Astrochemistry from Electron Attachment to NCCCCN. Astrophys. J. 2008, 685, L95-L98.

(16) Rajput, J.; Lammich, L.; Andersen, L. H. Measured Lifetime of $\mathrm{SF}_{6}^{-}$. Phys. Rev. Lett. 2008, 100, 153001.

(17) Chandrasekaran, V.; Prabhakaran, A.; Kafle, B.; Rubinstein, H.; Heber, O.; Rappaport, M.; Toker, Y.; Zajfman, D. Formation and stabilization of $\mathrm{C}_{6}^{-}$by radiative electron attachment. J. Chem. Phys. 2017, 146, 094302.

(18) Petrie, S. Novel pathways to $\mathrm{CN}^{-}$within interstellar clouds and circumstellar envelopes: implications for IS and CS chemistry. Mon. Not. R. Astron. Soc. 1996, 281, 137-144.

(19) Petrie, S.; Herbst, E. Some interstellar reactions involving electrons and neutral species: attachment and isomerization. Astrophys. J. 1997, 491, 210.

(20) Terzieva, R.; Herbst, E. Radiative electron attachment to small linear carbon clusters 
and its significance for the chemistry of diffuse interstellar clouds. Int. J. Mass Spectrom. 2000, 201, $135-142$.

(21) Herbst, E.; Osamura, Y. Calculations on the formation rates and mechanisms for $\mathrm{C}_{n} \mathrm{H}$ anions in interstellar and circumstellar media. Astrophys. J. 2008, 679, 1670.

(22) Douguet, N.; Fonseca dos Santos, S.; Raoult, M.; Dulieu, O.; Orel, A. E.; Kokoouline, V. Theory of radiative electron attachment to molecules: Benchmark study of $\mathrm{CN}^{-}$. Phys. Rev. A 2013, 88, 052710.

(23) Douguet, N.; Fonseca dos Santos, S.; Raoult, M.; Dulieu, O.; Orel, A. E.; Kokoouline, V. Theoretical study of radiative electron attachment to $\mathrm{CN}, \mathrm{C}_{2} \mathrm{H}$, and $\mathrm{C}_{4} \mathrm{H}$ radicals. $J$. Chem. Phys. 2015, 142, 234309.

(24) Khamesian, M.; Douguet, N.; Fonseca dos Santos, S.; Dulieu, O.; Raoult, M.; Brigg, W. J.; Kokoouline, V. Formation of $\mathrm{CN}^{-}, \mathrm{C}_{3} \mathrm{~N}^{-}$, and $\mathrm{C}_{5} \mathrm{~N}^{-}$molecules by radiative electron attachment and their destruction by photodetachment. Phys. Rev. Lett. 2016, 117, 123001.

(25) Kumar, S. S.; Hauser, D.; Jindra, R.; Best, T.; Roučka, S.; Geppert, W. D.; Millar, T. J.; Wester, R. Photodetachment as a destruction Mechanism for $\mathrm{CN}^{-}$and $\mathrm{C}_{3} \mathrm{~N}^{-}$anions in circumstellar envelopes. Astrophys. J. 2013, 776, 25.

(26) Pino, T.; Tulej, M.; Güthe, F.; Pachkov, M.; Maier, J. P. Photodetachment spectroscopy of the $\mathrm{C}_{2 n} \mathrm{H}^{-}(\mathrm{n}=2-4)$ anions in the vicinity of their electron detachment threshold. $J$. Chem. Phys. 2002, 116, 6126-6131.

(27) Best, T.; Otto, R.; Trippel, S.; Hlavenka, P.; von Zastrow, A.; Eisenbach, S.; Jézouin, S.; Wester, R.; Vigren, E.; Hamberg, M.; Geppert, W. D. Absolute photodetachment crosssection measurements for hydrocarbon chain anions. Astrophys. J. 2011, 742, 63. 
(28) Oana, C. M.; Krylov, A. I. Cross sections and photoelectron angular distributions in photodetachment from negative ions using equation-of-motion coupled-cluster Dyson orbitals. J. Chem. Phys. 2009, 131, 124114.

(29) Douguet, N.; Kokoouline, V.; Orel, A. E. Photodetachment cross sections of the $\mathrm{C}_{2 n} \mathrm{H}^{-}(n=1-3)$ hydrocarbon-chain anions. Phys. Rev. A 2014, 90, 063410.

(30) Lara-Moreno, M.; Stoecklin, T.; Halvick, P.; Loison, J. C. Single-center approach for photodetachment and radiative electron attachment: Comparison with other theoretical approaches and with experimental photodetachment data. Phys. Rev. A 2019, 99, 033412 .

(31) Heays, A. N.; Bosman, A. D.; van Dishoeck, E. F. Photodissociation and photoionisation of atoms and molecules of astrophysical interest. Astron. Astrophys. 2017, 602, A105.

(32) Draine, B. T. Photoelectric heating of interstellar gas. Astrophys. J. Suppl. S. 1978, $36,595-619$.

(33) van Dishoeck, E. F.; Black, J. H. The excitation of interstellar C2. Astrophys. J. 1982, $258,533-547$.

(34) Light, J. C.; Ross, J.; Shuler, K. E. Rate coefficients, reaction cross sections and microscopic reversibility. Kinetic processes in gases and plasmas. 1969; pp 281-320.

(35) Milne, E. XVIII. Statistical equilibrium in relation to the photo-electric effect, and its application to the determination of absorption coefficients. Phil. Mag. Series 1924, 47, 209-241.

(36) Tully, J. C.; Berry, R. S.; Dalton, B. J. Angular distribution of molecular photoelectrons. Phys. Rev. 1968, 176, 95-105. 
(37) Reed, K. J.; Zimmerman, A. H.; Andersen, H. C.; Brauman, J. I. Cross sections for photodetachment of electrons from negative ions near threshold. J. Chem. Phys. 1976, $64,1368-1375$.

(38) Grell, G.; Bokarev, S. I.; Winter, B.; Seidel, R.; Aziz, E. F.; Aziz, S. G.; Kühn, O. Multi-reference approach to the calculation of photoelectron spectra including spinorbit coupling. J. Chem. Phys. 2015, 143, 074104.

(39) Ponzi, A.; Angeli, C.; Cimiraglia, R.; Coriani, S.; Decleva, P. Dynamical photoionization observables of the CS molecule: The role of electron correlation. J. Chem. Phys. 2014, $140,204304$.

(40) Werner, H.-J.; Knowles, P. J.; Knizia, G.; Manby, F. R.; Schütz, M., et al. MOLPRO, version 2012.1, a package of ab initio programs. 2012; see http://www.molpro.net.

(41) Millar, T. J.; Walsh, C.; Cordiner, M. A.; Chuimín, R. N.; Herbst, E. Hydrocarbon anions in interstellar clouds and circumstellar envelopes. Astrophys. J. 2007, 662, L87.

(42) Millar, T. J.; Walsh, C.; Cordiner, M. A.; Chuimín, R. N.; Herbst, E. Hydrocarbon Anions in Interstellar Clouds and Circumstellar Envelopes. Astrophys. J. 2007, 662, L87-L90.

(43) Walsh, C.; Harada, N.; Herbst, E.; Millar, T. J. The effects of molecular anions on the chemistry of dark clouds. Astrophys. J. 2009, 700, 752-761.

(44) Wigner, E. P. On the behavior of cross sections near thresholds. Phys. Rev. 1948, 73, $1002-1009$.

(45) Cordiner, M. A.; Millar, T. J. Density-enhanced gas and dust shells in a new chemical model for IRC +10206. Astrophys. J. 2009, 697, 68-78.

(46) Desfrançois, C.; Abdoul-Carime, H.; Schermann, J.-P. Ground-state dipole-bound anions. Int. J. Mod. Phys. B 1996, 10, 1339-1395. 


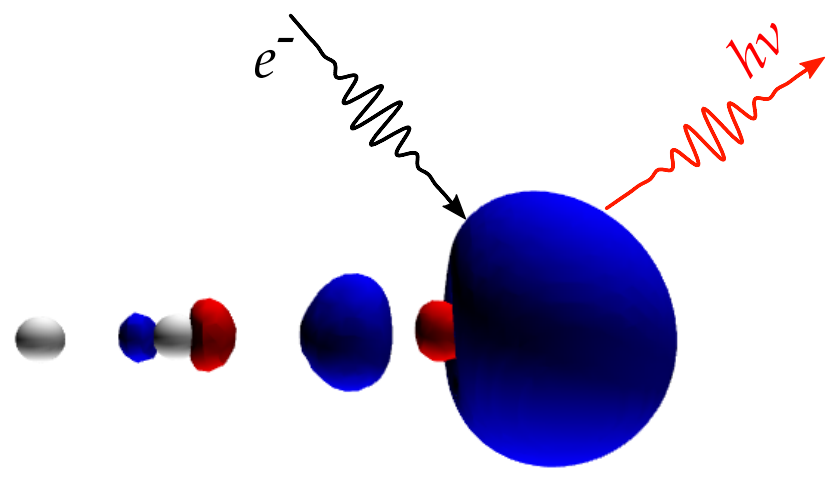

Figure 3: For TOC only. 